\title{
Non-rigid 2D-3D Registration with Catheter Tip EM Tracking for Patient Specific Bronchoscope Simulation
}

\author{
Fani Deligianni, Adrian J. Chung, and Guang-Zhong Yang \\ Department of Computing, Imperial College London \\ \{fani.deligianni, ajchung, g.z.yang\}@imperial.ac.uk \\ http://www.doc.ic.ac.uk/ gzy/vis-a-ve
}

\begin{abstract}
This paper investigates the use of Active Shape Models (ASM) to capture the variability of the intra-thoracic airway tree. The method significantly reduces the dimensionality of the non-rigid $2 \mathrm{D} / 3 \mathrm{D}$ registration problem and leads to a rapid and robust registration framework. In this study, EM tracking data has been also incorporated through a probabilistic framework for providing a statistically optimal pose given both the EM and the image-based registration measurements. Comprehensive phantom experiments have been conducted to assess the key numerical factors involved in using catheter tip EM tracking for deformable 2D/3D registration.
\end{abstract}

\section{Introduction}

The clinical practice of minimal invasive surgery has advanced rapidly in recent years due to improved therapeutic outcome coupled with reduced patient trauma and hospitalization. The technique, however, requires extensive manual dexterity and hand-eye coordination due to the complexity of instrument control and a loss of 3D vision and tactile feedback. In bronchoscope, for example, these skills are typically acquired based on the apprentice scheme or inanimate plastic models. The use of high-fidelity simulators provides an attractive alternative which offers a more complete training curriculum by incorporating usual, as well as rare pathological cases at a significantly lower cost and without the inference of routine patient care.

The prerequisite of constructing high-fidelity simulation models is the provision of visually realistic rendering based on patient-specific biomechanical models to recreate an environment that is close to real surgical scenes. This approach of patient specific simulation has attracted considerable interests in recent years [1,2], but photorealism and biomechanical fidelity remain active research topics [3-5]. In bronchoscope simulation, an essential requirement for photorealistic rendering and modeling is accurate $2 \mathrm{D} / 3 \mathrm{D}$ registration of the $2 \mathrm{D}$ bronchoscopy video with $3 \mathrm{D}$ tomographic data. Despite the success of rigid 2D/3D registration achieved in recent years, existing research has shown that the accuracy of the current registration techniques is compromised due to the deformation of the airways [6]. During respiration, the intra-thoracic tree is affected by both respiratory and cardiac motion and it undergoes significant anatomical changes even during normal breathing. This explains why most 
of the current techniques become problematic in registering distal airways where structural deformation is at its greatest.

The incorporation of airway deformation to the existing 2D/3D registration framework, however, is by no means trivial. This is due to the potentially large search space involved in simultaneous tracking of the camera pose and morphological deformations. Issues concerning mesh folding and the sensitivity of the similarity function to lighting and local minima represent a major challenge. With the recent advent of catheter tip EM trackers, research is now directed towards using the device during the bronchoscope procedure to simplify the $2 \mathrm{D} / 3 \mathrm{D}$ registration process [7]. These devices typically have 5 degrees-of-freedom (DoF) and have also been used in a number of image-guided applications such as for precision biopsy [8] and spine surgery [9].

As a first glance, it may seem that the use of $5 \mathrm{DoF}$ catheter tip EM tracker reduces the original $2 \mathrm{D} / 3 \mathrm{D}$ registration to only a $1 \mathrm{D}$ problem. It has been postulated that in the presence of airway deformation, one can readily use the EM tracking result to recover the unknown axial camera rotation [10]. After careful in vivo experiment, however, the true extent of the problem has been recognized and it has been found that the effect of respiratory motion in previous studies has not been fully appreciated. This is because the EM tracker reports the position and orientation of the bronchoscope camera in relation to the fixed, world coordinates. When this information is used to guide the registration algorithm to the static airway acquired from tomographic data, a large displacement due to airway deformation can be observed. When this EM tracking information is used to initialize the $2 \mathrm{D} / 3 \mathrm{D}$ registration, it has often been found that the initial view can be completely outside of the bronchial tree. This initialization is in fact inferior to the traditional image based approach, as in this case the camera view is always in synchrony with the moving frame of reference of the deforming airways. For this reason, direct mapping of the EM tracking measurements to the static pre-operative CT images is not be appropriate, and its practical use must be integrated with the deformation of the airways.

The purpose of this paper is to examine the use of Active Shape Model (ASM) to capture the variability of the intra-thoracic tree. In this way, it is expected that the dimensionality of the non-rigid 2D-3D registration problem can be significantly reduced, thus leading to a rapid and robust registration framework. Since it is difficult to obtain the ground truth for in vivo data, this study is focused on the use of comprehensive phantom validation to assess the key numerical factors involved in using catheter tip EM tracking for deformable 2D/3D registration.

\section{Methods}

\subsection{Modeling the Respiratory Motion of the Intra-thoracic Tree}

Physically-based models are controlled by the laws of motion dynamics and corresponding tissue properties. They generally result in accurate bio-mechanical representation of the deformation. However, the practical implementation of this approach can be difficult due to the lack of detailed tissue mechanical properties, as 
well as the prohibitive computational cost involved. One alternative is to use general shape modeling such as the NURBS based surface representation for the intrathoracic tree [5]. In addition to the issue of biomechanical fidelity, the main disadvantage of this approach is the mathematical complexity it introduces and the large number of unrestricted parameters that control the shape of the NURBS.

In this study, the ASM is used to provide subject specific modeling of the deformation of the airways during respiration. ASM provides an effective means of capturing statistically plausible variations of an object shape through a number of observations [11]. ASM is based on Point Distribution Models (PDMs), where point correspondence between a set of registered images is established and the variability of the shape can be expressed through a set of a linear combination of the mean shape $\bar{s}$ derived from the training set and its modes of variation. That is,

$$
s=\bar{s}+P b
$$

where $P$ is the eigenvectors and $b$ the parameter that varies to create new shapes that are restricted by the main modes of variation. With ASM, simplification of the model is possible by selecting only the principal modes of variation. The first mode of variation, for example, typically describes the main motion of the tracheobronchial tree due to respiration, whereas other modes are related to high-order local variabilities.

It is important to note that the success of ASM depends on the number and quality of correspondent landmarks across the training shapes. To establish correspondence between a set of control points across the $3 \mathrm{D}$ meshes, a semi-automatic method was used. The skeleton of the airway tree was first extracted from the $3 \mathrm{D} \mathrm{CT}$ data. The bifurcations of the airway tree were then used as the landmarks for establishing shape correspondences. For each of the skeleton points, the surface perpendicular to the skeleton axis was defined and its intersection with the 3D mesh was estimated. The application of ASM to the skeletal and surface control points results in intermediate, deformed airway structures. To generate a smooth 3D mesh of the airways, Radial Basis Functions (RBFs) are used. RBFs provide an easily controllable behaviour based on a sparse and irregular set of control points and thus they are suitable for deforming the tracheo-bronchial mesh.

\subsection{Incorporating Temporal Correspondence}

The use of ASM allows the incorporation of deformable modeling into the registration process. To further improve the registration accuracy, temporal tracking based on the Condensation algorithm has been used. Particularly, the condensation algorithm permits the use of multi-modal probability distributions that is essential to track the camera position under airway deformation and image artifacts [12]. Mathematically, the condensation algorithm does not explicitly represent the density function. It uses instead statistical factor sampling based on the Bayesian theory. During statistical factored sampling, a sample set is generated from the prior density. A weight is subsequently assigned to each particle according to the observation density. The weighted point set is then used as a representation of the a posteriori density. Subsequently, by evaluating the given moments of the state density, it is possible to estimate the most probable state. During prediction, the method involves the evolution of the state vector with time. Sampling methods based on spatial 
Markov processes are generally used for the Condensation method to represent the dynamics of a moving camera.

For the purpose of this study, the observation/measurement model needs to combine both the EM tracker measurements and the image-based registration results. Generally, the observation density $p\left(z_{t} \mid x_{t}\right)$ defines the likelihood that a state $x_{t}$ causes the measurement $z_{t}$. This is typically achieved by assuming that the measurements are statistically independent both mutually and with respect to the process model. Furthermore, both the EM tracker and the image-based registration measurements are imprecise. In this study, the uncertainty is quantified with a Gaussian probability density function $p \sim \exp (0, \sigma)$, where the variance $\sigma$, depends on the reliability of the different sensors measurements. The observation density for a particular state is estimated by integrating over the space of sensors measurements:

$$
p\left(z_{t} \mid x_{t}\right)=\sum_{m} p_{z_{t}^{m}}
$$

For 2D/3D registration, a $p q$-space based 2D/3D registration technique developed by Deligianni et al has been used [1]. The state of the system has been defined as the $6 \mathrm{DoF}$ pose of the camera. However, since EM tracking data are available only for 5 out of $6 \mathrm{DoF}$, the last degree of freedom is tracked independently.

Finally, for the process model, a second order auto-regressive model was used to describe the motion of the endoscopic camera [13]. The auto-regressive model takes into account that during bronchoscope navigation, motion occurs within a bounded area, and a rapidly moving camera is expected to slow down or change in direction rather than accelerate further. A second-order auto-regressive model is described by the following equation:

$$
x_{t}=A_{1} x_{t-1}+A_{2} x_{t-2}+B w t
$$

where $A_{k}$ represents the collection of damped harmonic oscillators associated with vibrational modes and $w$ the white noise with covariance coefficient $B$. This ensures smooth navigation of the camera. With modular training, multiple training sets can be used to obtain a more general model of the motion behavior .

Modular training facilitates the incorporation of multiple sequences from different subjects as well as the incorporation of the dynamic behavior of camera navigation in different parts of the tracheo-bronchial tree [13, 14]. Based on the principles of the maximum likelihood learning, the parameters of the second order autoregressive model can be estimated. The motion characteristics such as the coefficients $A_{k}$, the mean value $\bar{X}$ of the state and the random component $B$ are obtained from a number of different training sequences derived directly from the 5 DoF EM data, while for the sixth degree of freedom manually collected data has been used.

\subsection{Validation}

To validate the proposed technique, a deformable airway phantom made of silicon rubber and painted with acrylics was used. The inner surface was coated with silicon-rubber mixed with acrylic to give it a textured specular finish that is similar to 
the bronchial lumen. The phantom surface with finished with red color splashes to imitate the blood and texture discrepancies during a bronchoscope examination. The distal ends of the airway-phantom have been fixed and a balloon was located below the main bifurcation to simulate respiratory induced the airway deformation. The extent of the airway motion is regulated by a valve to control the amount of air in the balloon. The tomographic model of the phantom was scanned with a Siemens Somaton Volume Zoom 4-channel multi-detector CT scanner with a slice thickness of $1 \mathrm{~mm}$ and in-plane resolution of $1 \mathrm{~mm}$. A total of six $C T$ volumes were acquired at different stages of the motion cycle. An Olympus bronchoscope (Olympus BF Type, with a field of view $120^{\circ}$ ) operating in PAL recording mode (25fps) was used to capture the $2 \mathrm{D}$ bronchoscope video. Prior to 2D/3D registration, pre-processing of the videos was necessary in order to alleviate the effects of interlacing, lens distortion and image artefacts [15].

To track the pose of the bronchoscope camera, an NDI (Northern Digital Inc, Ontario) Aurora EM tracker is used. The Aurora system is particularly suited to tracking flexible endoscopes during minimally invasive intervention. This typically employs a single coil sensor due to size restrictions imposed by the dimensions of the biopsy channel or catheter. Having only a single coil, the sensor is limited to reporting position and direction only (i.e. 5 DoF with unknown axial rotation). The working volume of the Aurora system is a $500 \mathrm{~mm}$ cube located $50 \mathrm{~mm}$ from the field generator. In an environment that is free from electromagnetic interference, the Aurora has a static positional accuracy of $0.9-1.7 \mathrm{~mm}$ and an orientation accuracy of 0.5 degree .

In order to provide the ground truth data for airway deformation, a 6 DoF EM tracker was used to track the deformation of the balloon so that the deformation of the phantom airway can be indexed back to the 3D CT data. Based on the assumption that these points are moving in parallel for each positional value of the 6 DoF EM tracker the phase of the respiratory motion can be estimated as:

$$
b_{1}=\vec{M} \cdot p_{r} \cdot\left(p_{r} \cdot p_{r}^{T}\right)^{-1}
$$

where $p_{r}$ is the eigenvector that corresponds to the point $s_{r}$ of the mean shape that is nearest to the position of the 6DoF EM tracker and $\vec{M}$ is the vector between these points.

\section{Results}

In Fig. 1 the recovered deformation from the ASM model is presented. During 2D/3D registration, this has been used to directly deform the tracheo-bronchial mesh by tuning the first principal component of the ASM. The markers point out the location of the frames depicted in Fig. 2, which provides a qualitative validation of the recovered coefficients of the ASM. In this figure, column (a) represents the original bronchoscope video frames and column (b) illustrates the rendering pose by directly using the EM data. It is evident that the position of the camera changes rapidly due to the respiratory motion in the world coordinates, and a direct use of the EM data can result in initial camera position located completely outside of the bronchial tree. The result of incorporating $\mathrm{ASM}$ to the $2 \mathrm{D} / 3 \mathrm{D}$ registration framework is shown in Fig. 2(c), which defines the position and orientation of the camera such that the 
remaining axial rotation can be recovered as shown in Fig. 2(d) . Finally, Fig. 2(e) demonstrates the results by using Fig. 2(d) to initialize the 6 DoF $p q$-based registration [1].

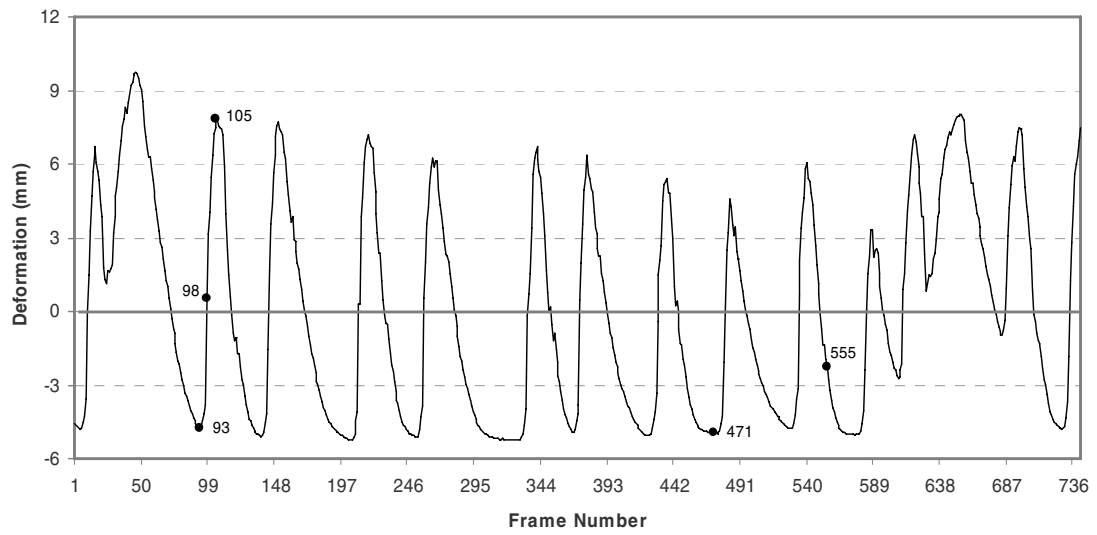

Fig. 1. Recovered deformation that can be used to directly deform the tracheo-bronchial mesh by tuning the first principal component of the ASM

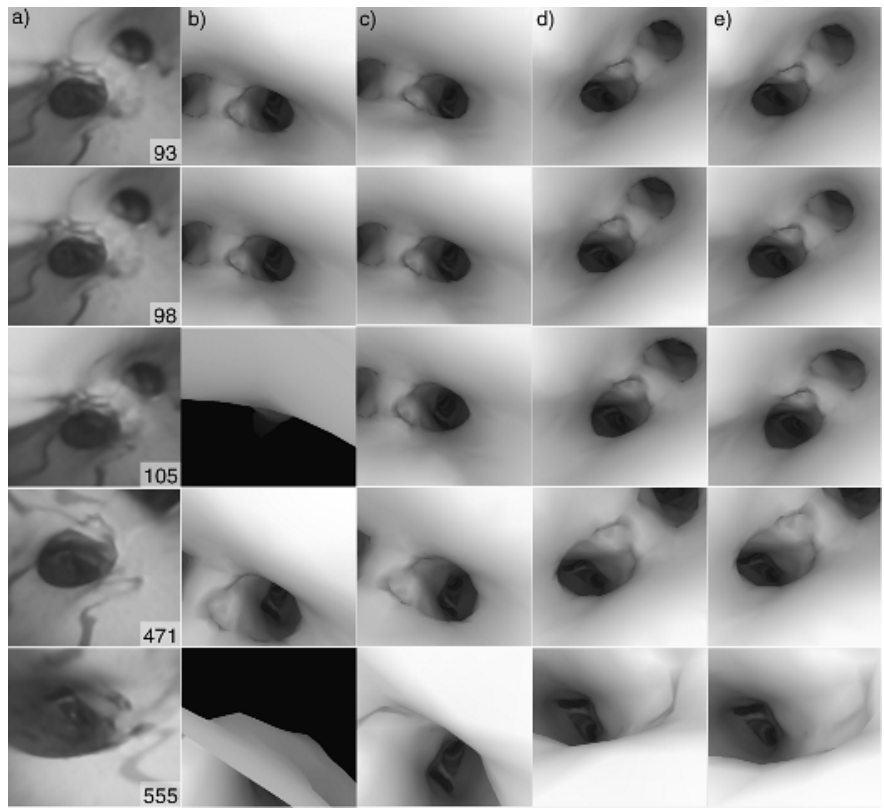

Fig. 2. Example bronchoscope video frames (a), and the corresponding registration results by a direct application of the EM tracking information (b), and by deformation modeling with ASM (c). Columns (d)-(e) illustrates the registration results after axial rotation recovery and subsequent 6 DoF $p q$-based registration, respectively 


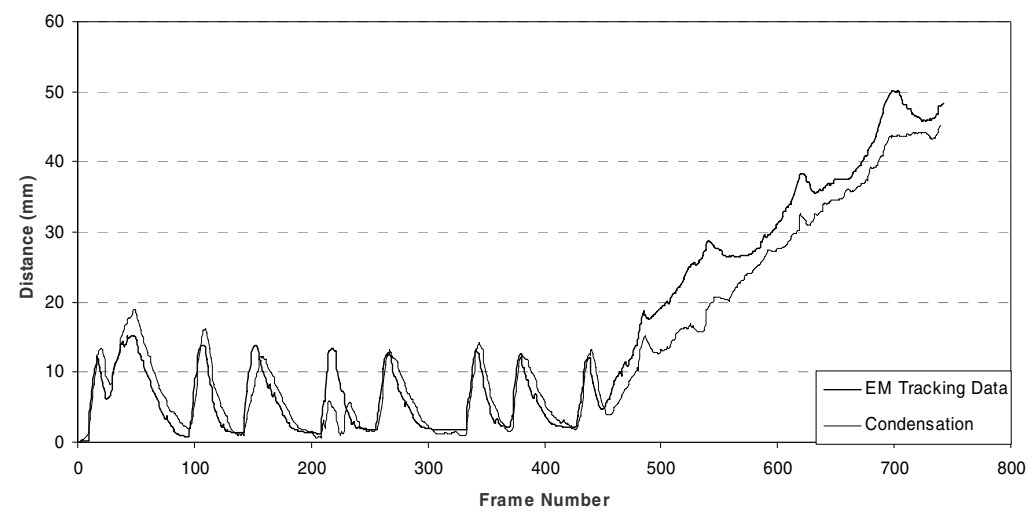

Fig. 3. Euclidean distance between the first and subsequent camera position as it has been predicted from the Condensation algorithm with relation to the EM tracking data

To provide a quantitative measure of the tracking algorithm described above, Fig. 3 illustrates the results by applying the Condensation algorithm the entire video to segment as compared to the ground truth data recovered from the EM data. Temporal tracking incorporates both the measurements of the EM tracker andc the image-based registration results, and thus it is able to recover when either of them has failed.

\section{Discussion and Conclusions}

Despite the prevalence of tissue deformation in most surgical navigation and planning applications, its incorporation into the 2D/3D registration framework has been limited. This is due to the large degrees of freedom involved in the registration parameters, which make a stable implementation of the algorithm difficult. In this paper, we have developed a non-rigid 2D/3D registration framework that models the respiratory motion of the intra-thoracic tree based on ASM. ASM captures the intrinsic variability of the airways across different phases of the respiratory motion and it constrains the motion they represent to be specific to the subject studied. This allows the subsequent non-rigid registration implemented in a much reduced dimensional space, and thus greatly simplifies the 2D/3D registration procedure that incorporates temporal correspondence and local adjustment. The detailed phantom validation of the method in this study demonstrates the potential clinical value of the technique.

\section{References}

1. Deligianni F, Chung A, Yang GZ. Patient-Specific Bronchoscope Simulation with pqSpace-Based 2D/3D Registration. Computer Aided Surgery 2004; 9(5): 215-226.

2. Chung AJ, Deligianni F, Shah P, Wells A, Yang GZ. Vis-a-Ve: Visual Augmentation for Virtual Environments in Surgical Training. in Proceedings of Eurographics / IEEE VGTC Symposium on Visualization, Leeds, UK, 2005, 101-108. 
3. Chung AJ, Deligianni F, Shah P, Wells A, Yang GZ. Patient Specific Bronchoscopy Visualisation through BRDF Estimation and Disocclusion Correction. IEEE Transactions of Medial Imaging (accepted for publication), 2005.

4. Deligianni F, Chung A, Yang GZ. Non-Rigid 2D/3D Registration for Patient Specific Bronchoscopy Simulation with Statistical Shape Modelling. IEEE Transactions on Medical Imaging (under review), 2005.

5. Garrity JM, Segars WP, Knisley SB, Tsui BMW. Development of a Dynamic Model for the Lung Lobes and Airway Tree in the Ncat Phantom. IEEE Transactions on Nuclear Science 2003 ; 50(3): 378-383.

6. Mori K, Deguchi D, Sugiyama J, Suenaga Y, Toriwaki J-i, Jr. CRM, Takabatake H, Natori H. Tracking of a Bronchoscope Using Epipolar Geometry Analysis and Intensity-Based Image Registration of Real and Virtual Endoscopic Images. Medical Image Analysis 2002; 6(3): 181-336.

7. Hautmann H, Schneider A, Pinkau T, Peltz F, Feussner H. Electromagnetic Catheter Navigation During Bronchoscopy: Validation of a Novel Method by Conventional Fluoroscopy. Chest 2005; 128(1): 382-387.

8. Banovac F, Glossop N, Lindisch D, Tanaka D, Levy E, Cleary K. Liver Tumor Biopsy in a Respiring Phantom with the Assistance of a Novel Electromagnetic Navigation Device. in Proceedings of Conference on Medical Image Computing and Computer Assisted Intervention (MICCAI02), Tokyo, Japan, 2002, 200-207.

9. Sagi HC, Manos R, Benz R, Ordway NR, Connolly PJ. Electromagnetic Field-Based Image-Guided Spine Surgery Part One: Results of a Cadaveric Study Evaluating Lumbar Pedicle Screw Placement. Spine 2003; 28(17): 2013-2018.

10. Mori K, Deguchi D, Akiyama K, Kitasaka T, Maurer CR, Suenaga Y, Takabatake H, Mori M, Natori H. Hybrid Bronchoscope Tracking Using a Magnetic Tracking Sensor and Image Registration. in Proceedings of International Conference on Medical Image Computing and Computer Assisted Intervention (MICCAI05), Palm Springs, California, USA, 2005, 543-550.

11. Cootes TF, Taylor CJ, Cooper DH, Graham J. Active Shape Models: Their Training and Application. Computer Vision and Image Understanding 1995; 61(1): 38-59.

12. Isard M, Blake A. Condensation-Conditional Density Propagation for Visual Tracking. International Journal of Computer Vision 1998; 29(1): 5-28.

13. Deligianni F, Chung A, Yang GZ. Predictive Camera Tracking for Bronchoscope Simulation with Condensation. in Proceedings of International Conference on Medical Image Computing and Computer Assisted Intervention (MICCAI05), Palm Springs, California, USA, 2005, 910-916.

14. North B, Blake A, Isard M, Rittscher J. Learning and Classification of Complex Dynamics. IEEE Transactions on Pattern Analysis and Machine Intelligence 2000; 22(9): 1016-1034.

15. Yang GZ, Burger P, Firmin DN, Underwood SR. Structure Adaptive Anisotropic Image Filtering. Image and Vision Computing 1994; 14(2): 135-145. 